\title{
Erdbülten als Frühjahrsformen im Raum um Göttingen
}

\author{
Von Dieter Kelletat, Göttingen
}

\author{
Mit 5 Abbildungen und 3 Tabellen
}

Zus a m menfassung. Es wird eine Beschreibung von Form, Verbreitung, Vegetationsbedeckung und innerem Bau von Erdbülten im Göttinger Raum gegeben. Die untersuchten Formen liegen bevorzugt auf künstlichen Böschungen ohne abgeschlossene Bodenentwicklung, von denen regelmäßig durch Grasbrände im Frühjahr die Vegetation entfernt wird. Kräftige Nachtfröste können dann zu einem Aufdringen von Bodenmaterial und zur Bildung der Erdbülten führen.

$\mathrm{Sum}$ m a ry. A description of form, distribution, vegetational cover, and build up of little hummocks is given. The investigated phenomena are situated especially on artificial slopes without finally soil development and with regular burning down of the vegetation in springtime. Following nightfrost can effect the rise of soil and the development of the hummocks.

\section{Einleitung}

Erdbülten als scharenweise auftretende Buckel- und Kuppenformen in einer Vegetationsdecke sind aus der alpinen und periglazialen Höhenstufe der Gebirge seit langem bekannt und beschrieben. Sie bestehen dort häufig aus einem aufgewölbten Lehmkern, in und über dem kryoturbate Störungen liegen, besonders an der Grenze zur deckenden Humusschicht (Elsasser 1967, Stingl 1969 u. a.). Aufgrund dieser kryoturbaten Erscheinungen nehmen FURRER (1955) und ElSASSER (1967) spätglaziale bzw. vorzeitliche Entstehung an, während Höllermann (1964) u. a. an die Möglichkeit einer rezenten Bildung denken.

Neuerdings sind den Erdbülten offenbar verwandte Erscheinungen mit sicher rezenter Anlage aus dem Saarland von Selzer (1959) und Hard (1964) beschrieben worden. Nach den Beobachtungen von Selzer (1959, S. 217 ff.) entstehen solche Formen, die er wegen des charakteristischen breiten Fußes und der aufragenden kleinen Erdpyramide als „Erdkegel“ bezeichnet, auf künstlichen, sehr mobilen Haldenböschungen durch artesischen Wasser- und Materialauftrieb an Schwächestellen eines massiven Frostbodens im Spätwinter oder zeitigen Frühjahr. Diese „Schlammdiapire“ frieren erst nachträglich und fallen durch Regen und Windwirkung im abgetrockneten Zustand sehr bald der Abtragung anheim.

Beobachtungen an rezenten Erdbülten als Frühjahrsformen im Göttinger Raum, deren Entstehung mit Sicherheit anders ist als an den von Selzer beschriebenen Formen scheinen dem Verfasser aufgrund ihrer weiten Verbreitung, ihrer auffälligen Gestalt und der Besonderheiten ihrer Lage einer kurzen Betrachtung wert.

\section{Die Formen der Erdbülten}

Die beigegebenen Abbildungen vermitteln einen Eindruck von der Vielgestaltigkeit der Bültenformen, welche häufig an einer Lokalität angetroffen werden können. Die Mehrzahl sind markante Erdkuppen, deren Höhe gleich oder gar größer als ihr Durchmesser an der Basis ist (Abb. 2-5). Schon von HARD (1964, S. 104) wird ausgeführt, daß die Blüten häufig asymmetrisch sind, an ihren Flanken verschieden dichten Bewuchs aufweisen können und nicht selten auch kahle Kuppen in Form einer „Tonsur" tragen. Diese kahlen Kuppen sind zudem oft abgeflacht (Abb. 1, Ziff. 3), offenbar durch Einwirkung von Regen u. a. etwas abgetragen. Einschnürungen oder Hohlkehlen an der hangabwärts gelegenen Basis wurden ebenfalls häufig beobachtet. Solche Formen, die im Verhältnis zu ihrem Durchmesser sehr hoch sind, sinken gelegentlich auch um, wobei die Richtung un- 
abhängig von der Exposition oder Hangneigung ist. Graswurzeln halten diese Gebilde noch aufrecht und wirken besonders bei schmalen Formen stabilisierend. Auf die Erscheinung, daß größeren breit entwickelten Bültenformen kleinere aufsitzen können, wies ebenfalls bereits HARD (1964, S. 104) hin.
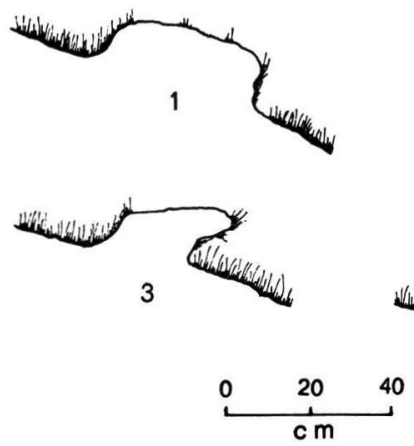

Abb. 1. Häufig auftretende Erdbültenformen im Gebiet um Göttingen.

Die Bültenformen um Göttingen treten zwar stets gesellig auf, doch liegen sie jeweils im Abstand von einigen Metern voneinander. Dieser Befund und die beschriebenen Formmerkmale deuten bereits einen auffälligen Unterschied zu den in regelrechten Bültenfeldern vergesellschafteten, sehr gleichartig gestalteten Buckeln alpiner Mattenregionen an. Nachfolgend seien einige Dimensionswerte aus einem Bültenfeld nördlich Oberscheden mitgeteilt.

Tabelle 1

Dimensionen von Erdbülten am Bahndamm nördlich Oberscheden a u f R öt (so). Höhenlage $240 \mathrm{~m}$ ü. NN, Exp. E, Böschungswinkel ca. $22^{\circ}$.

\begin{tabular}{c|c|c|c}
\hline $\begin{array}{c}\text { Höhe bergwärts } \\
\text { in cm }\end{array}$ & $\begin{array}{c}\text { Höhe talwärts } \\
\text { in cm }\end{array}$ & $\begin{array}{c}\text { Durchmesser in } \\
\text { halber Höhe, cm }\end{array}$ & weitere Merkmale \\
\hline 26 & 43 & 40 & kahle Kuppe \\
\hline 21 & 32 & 31 & \\
\hline 25 & 50 & 56 & kahle Kuppe \\
\hline 16 & 54 & 39 & 12 cm tiefe Hohlkehle \\
\hline 15 & 39 & 26 & hangabwärts überhängend \\
\hline 29 & 61 & 35 & kahle Kuppe \\
\hline 32 & 64 & 51 &
\end{tabular}

\section{Die Verbreitung der Erdbülten im Göttinger Raum}

Einige Lokalitäten mit besonders deutlich ausgebildeten Erdbülten gibt die folgende Aufstellung wieder: 
Tabelle 2

\begin{tabular}{|c|c|c|c|c|c|}
\hline Lokalität & Höhe ü. NN & Expos. & Hangneigung & Gestein & Standort \\
\hline $\begin{array}{l}\text { Leineberg, } \\
\text { Göttingen }\end{array}$ & $154 \mathrm{~m}$ & $\mathrm{E}$ & ca. $23^{\circ}$ & Löß/Rät & Bahnböschung \\
\hline $\begin{array}{l}\text { Hainberg, } \\
\text { Göttingen }\end{array}$ & $260 \mathrm{~m}$ & SW, S & $20-26^{\circ}$ & $\mathrm{mo}_{2}$ & Straßenböschung \\
\hline $\begin{array}{l}\text { E Jühnde, } \\
\text { N Endelskamp }\end{array}$ & $300 \mathrm{~m}$ & $\mathrm{~N}$ & $25-30^{\circ}$ & $\mathrm{mm}_{\mathrm{i}}$ & Straßenböschung \\
\hline $\begin{array}{l}\text { E Jühnde, } \\
\text { N Emmeberg }\end{array}$ & $235 \mathrm{~m}$ & $\mathrm{~N}, \mathrm{NE}$ & $0-8^{\circ}$ & $\mathrm{mu} / \mathrm{Löß}$ & Waldlichtung \\
\hline $\mathrm{N}$ Oberscheden & $240 \mathrm{~m}$ & W, E & $0-24^{\circ}$ & $\mathrm{so} / \mathrm{mu}$ & Bahnböschung \\
\hline (Altena/Westf.) & $(200 \mathrm{~m})$ & (SSE) & $\left(\right.$ ca. $\left.15^{\circ}\right)$ & (Tonschiefer) & $\begin{array}{l}\text { (aufgelassener } \\
\text { Garten) }\end{array}$ \\
\hline
\end{tabular}

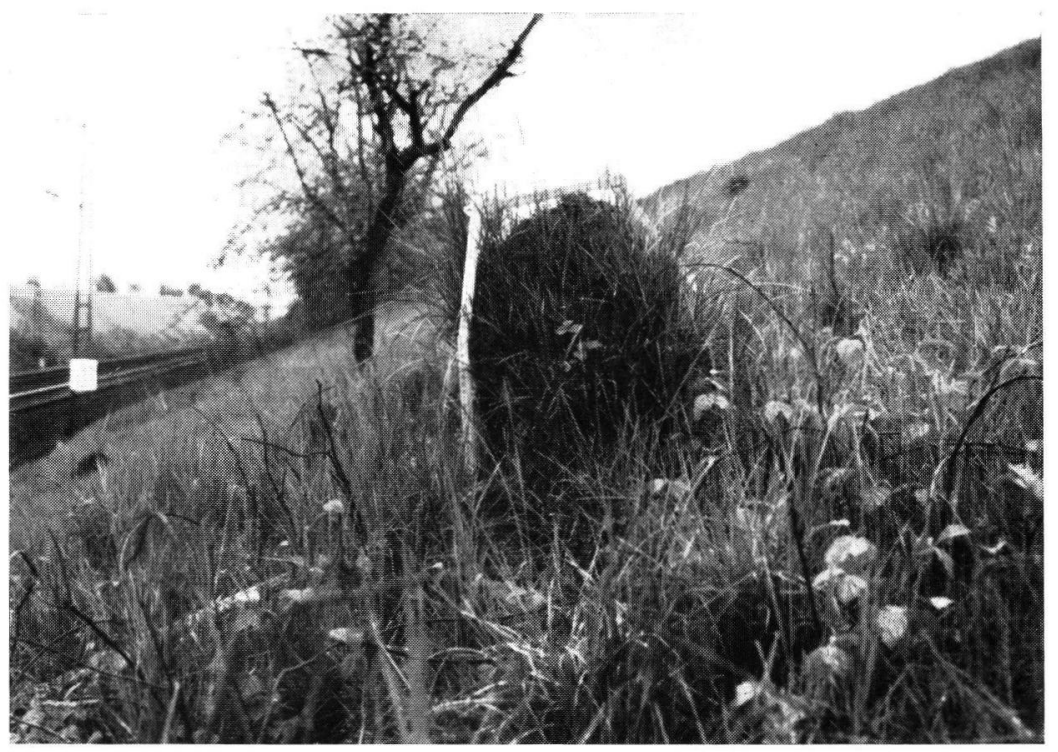

Abb. 2. Große Erdbülte auf einem ostexponierten, ca. $23^{\circ}$ geböschten Bahndamm am Leineberg (Göttingen). Aufnahme 13. 5. 1969.

Eine Abhängigkeit der Vorkommen von der Exposition ist nicht festzustellen. Es scheint solcher Untergrund bevorzugt zu sein, der ein sehr feinkörniges Verwitterungsprodukt liefert. Sehr auffällig ist das gehäufte Auftreten an mittelsteilen Böschungen. Als oberster Grenzwinkel wurde ca. $30^{\circ}$ beobachtet. Die Erdbülten wachsen auch an solchen Hängen immer senkrecht in die Höhe. Ganz selten konnte Verfasser Erdbülten bei Göttingen auch auf Ebenheiten finden. Das ist ein weiterer Gegensatz zu den aus den Alpen bekannten Vorkommen auf ebenen oder nur schwach geneigten Flächen.

Bezüglich des speziellen Standortes ergibt sich eine starke Übereinstimmung der jeweiligen Vorkommen, die noch durch eine Reihe weiterer, in Tabelle 2 nicht aufgeführter Beobachtungen gesichert werden konnte: Nahezu alle Erdbülten liegen auf künstlich geschaffenen oder veränderten Oberflächen, wenn diese auch teilweise schon einige Jahr- 
zehnte alt sein dürften. Gemeinsam ist ihnen eine noch nicht ausgeprägte Bodenbildung und -horizontierung. Auf allen oben angeführten Lokalitäten wird die Vegetation regelmäßig abgebrannt, so daß nur ein schwacher Wurzelfilz und eine oft schüttere Vegetationsdecke vorhanden ist. Alle Vorkommen liegen zudem ungehindert durch höhere Vegetation der direkten Ein- und Ausstrahlung ausgesetzt.

\section{Die Vegetationsbedeckung}

Die Erdbülten im Raum um Göttingen heben sich durch den unterschiedlichen Bewuchs von den umliegenden Hangteilen ab. Allerdings verwischt sich dieses Merkmal mit zum Sommer hin fortschreitender Vegetationsentwicklung. Selten nur wurde beobachtet, daß sich eine dichte Grasdecke mit abgestorbenen Pflanzenteilen älterer Jahre einige cm an den Flanken der Bülten hochzieht. Häufiger setzt sie am Fuß abrupt aus. In der Regel läßt sich dann noch ein junger (diesjähriger) Bewuchs von einer überwiegend kahlen Kuppe unterscheiden. Die Kahlstellen auf den Kuppen können asymmetrisch sein, doch wurden keine Beziehungen zur Exposition usw. festgestellt.

Nördlich des Emmeberges (im Osten von Jühnde) ist eine sehr große Erdbülte von ca. $1 \mathrm{~m}$ Höhe und Durchmesser neben kleineren ausgebildet (Abb. 3). Im Gegensatz zu den kleineren Formen der Umgebung und anderer Lokalitäten, die in der Pflanzenbesiedlung nur ein einziges junges Stadium erkennen lassen, konnten hier deutlich mindestens 3 verschiedene Bewuchszonen, von unten nach oben aufeinander folgend, festgestellt werden (Abb. 3, A-C). Eine untere Zone mit sehr dichtem Filz abgestorbener Graspflanzen läßt sich von einer höheren mit schwachem totem Pflanzenfilz und schließlich von einer obersten ohne Spuren eines älteren Bewuchses trennen.

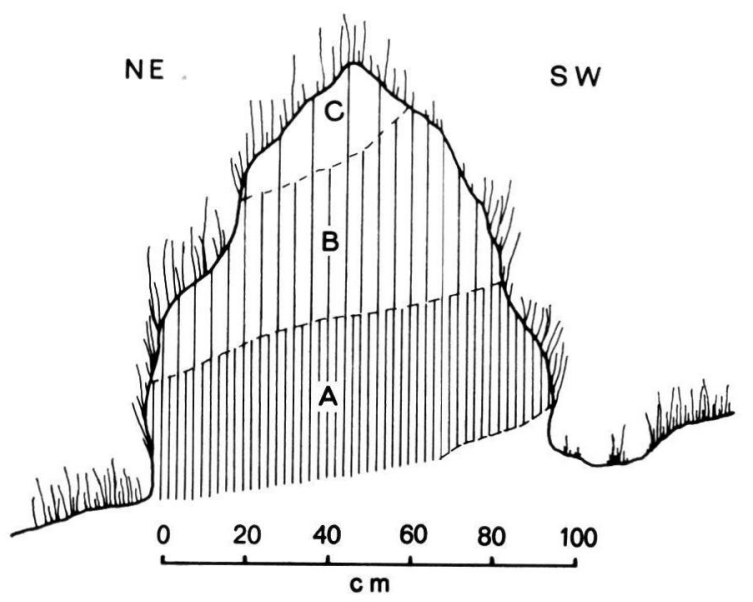

Abb. 3. Große Erdbülte nördlich des Emmeberges zwischen Jühnde und Obernjesa. Unter der diesjährigen Bewuchszone (C) liegt eine mindestens vorjährige (B), darunter eine noch ältere (A). Die Anlage der Form ist demnach 3 Jahre alt, der jüngste oberste Abschnitt entstand im Frühjahr 1969.

Die Vegetationsanordnung vermittelt so den Eindruck, als sei die große Erdbülte in 3 Phasen gewachsen, wobei die oberste jüngste Phase das diesjährige Stadium anzeigt. Häufig unterscheiden sich auch die kleinen, auf einen breiten Sockel aufgesetzten Bülten (Abb. 1, Ziff. 4) in gleicher Weise im Bewuchs von älteren Basisformen. Eine mehrphasige Entwicklung aufgrund des Bewuchses ist natürlich nur an solchen Stellen nachzuweisen, die nicht alljährlich abgebrannt werden. 


\section{Der innere Bau der Erdbülten um Göttingen}

Die kurze Zusammenstellung von Korngrößenanalysen aus alpinen Bültenböden bei ELSASSER (1967, S. 29) zeigt deutlich, daß diese überwiegend aus den stark frostgefährlichen Fraktionen 0,06-0,02 mm aufgebaut werden. Die Ergebnisse von 4 Proben aus einer Erdbülte am Leineberg in Göttingen (Löß/Rät) vermitteln ein ganz ähnliches Bild (Abb. 4 und Tab. 3).

Tabelle 3

Korngrößenanalysen a us einer Erdbülte am Leineberg, Göttingen (vgl. Abb. 4). Naßsiebung bis $0,063 \mathrm{~mm},<0,063 \mathrm{~mm}$ Hydrometeranalyse.

\begin{tabular}{c|c|c|c|c}
\hline \multirow{2}{*}{ Korngrößen in mm } & \multicolumn{4}{|c}{ Gewichtsprozent } \\
\cline { 2 - 5 } & Probe 1 & Probe 2 & Probe 3 & Probe 4 \\
\hline$>0,2$ & 7,8 & 5,6 & 3,8 & 1,3 \\
\hline $0,2-0,063$ & 13,1 & 8,5 & 7,8 & 8,7 \\
\hline $0,063-0,020$ & 58,0 & 48,3 & 57,0 & 54,2 \\
\hline $0,020-0,006$ & 15,4 & 29,1 & 23,2 & 29,2 \\
\hline$<0,006$ & 5,7 & 8,5 & 8,2 & 6,6 \\
\hline
\end{tabular}

Selzer (1959) betont, daß beim Aufgraben kein „Förderschlot“ des aufgedrungenen Materials sichtbar wurde, was damit zusammenhängt, daß bei den von ihm untersuchten Formen auf einer künstlichen Halde keine Bodenbildung vorliegt. HARD (1964) gelang es mit Hilfe des pH-Wertes nachzuweisen, daß Erdbülten aus nicht entkalktem Material bestanden, welches aus einer Tiefe von wenigen $\mathrm{dm}$ unterhalb eines weitgehend entkalkten Oberbodens gefördert worden sein muß. Der vertikale Transportweg maß dabei über $50 \mathrm{~cm}$.

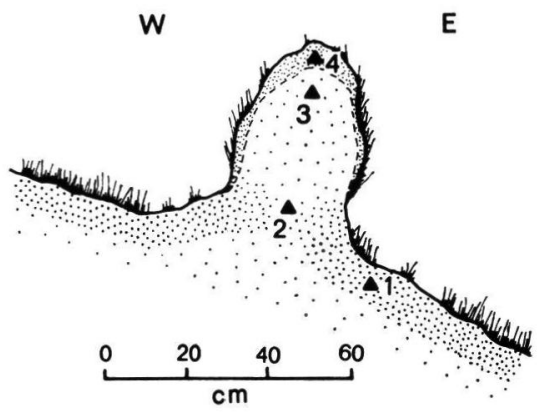

Abb. 4. Aufbau und Entnahmestellen der Proben (1-4) an einer Erdbülte am Leineberg (Göttingen) (vgl. Abb. 2).

1 - dunkler, humusreicher Oberboden, ca. $10 \mathrm{~cm}$ mächtig, nach unten unscharf begrenzt, oberflächlich viele Brandspuren.

2 - Mischzone des dunklen Oberbodens mit aufgedrungenem helleren Material, keine verbrannten Pflanzenteile.

3 - Kern aus lockerem, von Bodentieren durchwühltem Feinmaterial, mittelbraun, keine Brandspuren.

4 - gelblich-braunes, hellstes und leicht verfestigtes Feinmaterial ohne organische Substanzen. 
Grabungen an Erdbülten im Göttinger Raum ergaben einen charakteristischen Aufbau: Das Bültenmaterial ist durchweg heller als die danebenliegende oberste Bodenschicht, in der eine Humusanreicherung, ein Wurzelfilz und Spuren häufiger Grasbrände zu finden sind (Abb. 4, Probe 1). Einen durch Bodentiere mehr oder weniger gelockerten Kern (Probe 3) umgibt an den Flanken, besonders mächtig aber auf der Kuppe eine abgetrocknete, wurzelfreie und resistente Schicht ohne organische Substanzen.

Durch Grabungen ließ sich nachweisen, daß das Bültenmaterial aus einiger Tiefe unter dem dunkelgefärbten Oberboden stammen muß. Um in einem Anschnitt den „Förderschlot" (heller Kern mit dunklem Ring) freizulegen, muß man die Bülten oft einschließlich ihres eventuell vorhandenen Sockels kappen, da wenig höher durch die Mischung des aufgedrungenen Materials mit dem Oberboden (Abb. 4, Probe 2) keine eindeutigen Farbunterschiede festzustellen sind.

Im Gegensatz zu den Beobachtungen von Selzer (1959), der ein Aufdringen bis zu $10 \mathrm{~cm}$ großer Steine feststellte, fehlen den von mir aufgegrabenen Formen die Fraktionen größer als $1 \mathrm{~mm}$ auch an solchen Hängen, die größere Schuttstücke aufweisen.

\section{Hinweise zur Genese der Erdbülten im Göttinger Raum}

Die steilen Formen und der junge schüttere Bewuchs deuten auf ein sehr geringes Alter der Erdbülten im Göttinger Raum hin. Grabungen erbrachten den Nachweis, daß das Bültenmaterial aus einer Tiefe von wenigstens $10 \mathrm{~cm}$ unter der Oberfläche stammen muß. Eine ganze Reihe der in Tab. 2 aufgeführten Vorkommen sind innerhalb weniger Tage im Frühjahr 1969 (Altena/Westf. Frühjahr 1966) neu entstanden. Bereits SELzer (1959, S. 218) beobachtete ja im Frühjahr 1956, daß sich „Erdkegel“ von $60 \mathrm{~cm}$ Höhe in wenigen Tagen bilden können. Eine geringfügige Weiterentwicklung der Formen ist allerdings nicht auszuschließen.

In allen Fällen, in denen eine Neubildung der Erdbülten sicher beobachtet und datiert werden konnte, waren kurze Zeit vorher (Göttingen Ende März 1969, Altena 3. April 1966) die Grashänge abgebrannt worden, so daß die Vegetationsdecke und ein oberflächlicher Wurzelfilz zerstört waren. Nach etlichen Tagen warmer Witterung war der restliche Bodenfrost bereits völlig abgetaut und das Bodenmaterial stark durchfeuchtet (z. B. an Göttinger Wetterstation seit dem 23. 3. 1969 keine negativen Bodentemperaturen mehr). Setzten bei diesen Vorbedingungen kräftige nächtliche Kälterückfälle ein (in Göttingen Minimum am Erdboden $-3,9^{\circ} \mathrm{C}$ am 3. 4., $-6,2^{\circ} \mathrm{C}$ am 4. 4., $-4,7^{\circ} \mathrm{C}$ am 5. 4. 1969), so traten die Erdbülten schon am nächsten Morgen in aller Deutlichkeit auf den sonst ungegliederten abgebrannten Hängen als erdfarbene Kuppen auf schwarzem Untergrund hervor. Ihre Zahl konnte nach mehreren Frostnächten noch zunehmen.

In Altena/Westf. wurden nach einer Frostnacht am 6. 4. 1966 bei Aufgrabungen an Erdbülten Kerne gefrorenen Bodenmaterials gefunden, die sich zur Basis der Formen verbreiterten und in ca. $10 \mathrm{~cm}$ Tiefe unter der allgemeinen Hangfläche an unscharfer Grenze aussetzten. Bei direkter Bestrahlung um 11 Uhr lag der Kern des gefrorenen Bodens jeweils asymmetrisch an den beschatteten Flanken der Bülten (vgl. Abb. 5).

Nach diesen Beobachtungen und den Grabungsbefunden liegt es nahe, die Entstehung der Erdbülten auf nächtliches Gefrieren einer gut durchfeuchteten Bodenschicht aus stark frostgefährlichem Material der Fraktionen $0,06-0,02 \mathrm{~mm}$ zurückzuführen. Dabei steigt das Material offenbar an solchen Stellen besonders stark auf, wo durch vorheriges Abbrennen keine isolierende und hemmende Vegetationsdecke und Wurzelschicht mehr liegt. Die Entstehung nahezu aller Formen Ende März/Anfang April zeigt an, daß sie nicht notwendigerweise an massiven Bodenfrost gebunden sind. Der Unterschied zu den von SElzer (1959) beobachteten und beschriebenen Erdkegeln besteht also darin, daß die Formen als Frostbeulen wachsen, und nicht ungefrorenes Material diapirartig an Schwä- 


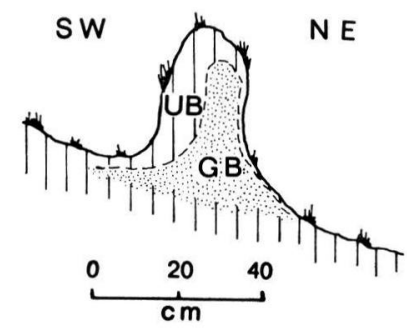

Abb. 5. Erdbülte mit einem Kern gefrorenen Bodens (GB) an der beschatteten Flanke bei Altena/ Westf. - Zustand vom 6. 4. 1966, 11 h. Grenze zum ungefrorenen Boden (UB) an der Basis unscharf. Der umliegende Hang war zum Zeitpunkt der Grabung ungefroren.

chezonen eines massiven Frostbodens aufdringt und erst nachträglich gefriert. Durch die unterschiedliche Genese dürften sich auch zwanglos die Formenunterschiede (Kegel mit breiter Basis bei SELzER im Gegensatz zu oft kolbenförmigen steilen Bülten bei Göttingen) erklären. Die relativ schwachen Frostkräfte können wohl kurzperiodisch nur dort ansetzen, wo die Wurzel- und Pflanzendecke durch den Menschen beseitigt wurde. Das gilt auch für das Vorkommen an der Waldlichtung des Emmeberges bei Jühnde, wo Spuren künstlicher Gräben und Gruben zu finden sind.

\section{Schlußbemerkungen}

Mit den vorstehenden Ausführungen mag ein Hinweis darauf gegeben sein, daß schon bei geringen Frostkräften die Entstehung markanter Bültenformen in kurzer Zeit möglich ist, und die Frage nach dem rezenten Alter ähnlicher alpiner Erscheinungen sicherlich nicht aus klimatischen Gründen verneint werden kann. Einen Unterschied zu den alpinen Gebilden belegt die viel größere Formenfülle bei Bültenböden im Göttinger Raum, ihr großer Abstand zueinander bzw. ihre jeweils isolierte Lage und ihre recht geringe Lebensdauer (in der Regel etwa bis zum nächsten Winter) sowie die enge Bindung an künstliche Böschungen mit stark behindertem Pflanzenwuchs und fehlender Bodenentwicklung. Eine Gemeinsamkeit besteht jedoch hinsichtlich der Beteiligung stark frostgefährlicher Korngrößen am Aufbau sowie der Häufung auch alpiner Vorkommen in der Nähe landwirtschaftlicher Gebäude u. ä. (Höllermann 1967, S. 22), wo ebenfalls eine gelegentliche Verletzung der isolierenden und schützenden Pflanzendecke gegeben sein dürfte.

Herrn E. SchröDER (Göttingen) danke ich für seinen Rat bei der Behandlung der Proben und der Wetterstation Göttingen für die Einsicht in ihre meteorologischen Meßergebnisse.

\section{L i t e r a $\mathrm{tr}$}

Elsasser, H.: Untersuchungen an Erdbülten im Avers. - Geogr. Helv. 22, Nr. 1, 27-30, Bern 1967.

Furrer, G.: Bodenformen aus dem subnivalen Bereich. - Die Alpen XXXI, Bern 1955.

HARD, G.: Noch einmal „Erdkegel“. - Eiszeitalter u. Gegenwart, 15, 102-107, O'Ohringen 1964. Höllermann, P.: Rezente Verwitterung, Abtragung und Formenschatz in den Zentralalpen am Beispiel des oberen Suldentales (Ortlergruppe). - Zeitschr. f. Geomorph. N. F., Suppl.-Bd. 4, Berlin 1964.

- : Zur Verbreitung rezenter periglazialer Kleinformen in den Pyrenäen und Ostalpen. — Gött. Geogr. Abh., 40, Göttingen 1967.

Selzer, G.: „Erdkegel“ als heutige Frostboden - Bildungen an Rutschhängen im Saarland. Eiszeitalter u. Gegenwart, 10, 217-223, Öhringen 1959.

Stingl, H.: Ein periglazialmorphologisches Nordsüdprofil durch die Ostalpen. - Gött. Geogr. Abh., 49, Göttingen 1969. 